\title{
Vibrations in space as an artificial gravitụ?
}

Daniel Beysens,

Service des Basses Températures, CEA-Grenoble, Grenoble (France) and Laboratoire de Physique et Mécanique des Milieux Hétérogènes, Ecole Supérieure de Physique et Chimie Industrielle•10, rue Vauquelin·75231 Paris Cedex 05 •France•E-mail: daniel.beysens@cea.fr

$\mathrm{T}$ he environment for satellites and spacecraft in space is markedly different from the terrestrial situation. Apart from the high vacuum, low temperature and intense external radiation environment, gravitational effects are absent. A wide variety of fundamental processes involving fluids and living systems are drastically affected; one of the major reasons being the absence of buoyancy flows (Fig. 1)

One way to improve the management of fluids and that of life support systems would be to create an "artificial" gravity. Classically, an artificial gravity can be produced in space in two ways. A centrifugal force, created by spinning the spacecraft or space station, can be used. However, large Coriolis forces are also present and everything would fall in curves instead of straight lines. One can also linearly accelerate the spaceships, but this cannot be continued over long periods because of the enormous energy costs. Less known would be the possibility to produce a uniform force field as coming from a magnetic field gradient, but here also the cost in energy is very high. (However, this solution can be advantageously used to compensate gravity on Earth as described below, see Box above fig. 4).

\section{Fluids and vibrations}

If we narrow our focus to fluid systems and to some extent to living organisms, vibrations provide another possible means of reproducing some of the effects of gravity. First of all, large amplitude (with respect to the system size), low frequency (with respect to the system time response) vibration gives a periodic acceleration - and then a periodic artificial gravity - to any entity. In order to illustrate this fact by an example, Fig. 2a shows a sample of supercritical fluid $\mathrm{CO}_{2}$ heated on Earth by a point source (a thermistor). It results in the convection of the less dense hot fluid parallel to gravity and an accumulation of hot fluid at the top, with a hot-cold interface perpendicular to gravity. The same phenomenon in space conditions and under vibration gives a similar convection pattern that is symmetrical with respect to the direction of vibration (Fig. 2b).

At small amplitude and high frequency, the situation is more complex and, at the same time, more interesting. In addition to local vibrations of the fluid, average flows are created. These flows

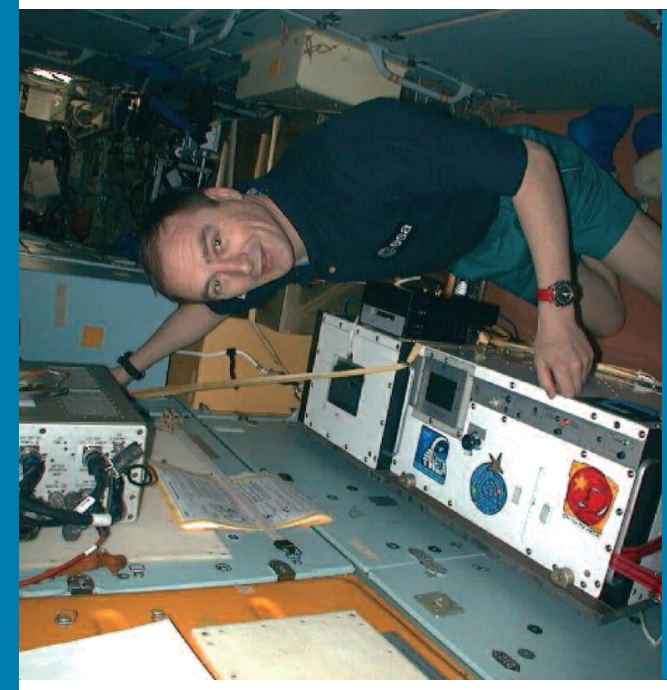

4 Fig. 1: The

conditions of weightlessness in space complicate and make

hazardous the management of fluids in satellites and spacecraft as well as the survival of human beings and, more generally, of living systems (J.P. Heigneré in the MIR station, 1999). are of inertial origin; they are connected to the nonlinear response of the fluid to the vibration and are initiated because of the existence of density inhomogeneities $\Delta \rho$. Average flows can also originate in the viscous hydrodynamic boundary layers at the cell walls or inclusions from where they propagate to the whole sample.

When submitted to a simple harmonic linear vibration $\mathrm{X}=\mathrm{acos} \omega \mathrm{t}$, a density inhomogeneity $\Delta \rho$ in a fluid of mean density $\rho$ can thus acquire a velocity difference $\Delta V=(\Delta \rho / \rho)$ a $\omega$. Contrary to the effect of vibrations of large amplitude and low frequency that direct inhomogeneities parallel to the direction of vibration, the low amplitude and high frequency vibration induces, in general, mean flows perpendicular to the vibration direction, as discussed below.

As a matter of fact, it is well known since the work of G.H. Wolf [1] that a gas-liquid interface subject to a horizontal vibration progressively orientates itself parallel to gravity. Simple reasoning, based on the presence of a Bernoulli pressure difference, gives a qualitative picture of the onset of motion at the beginning of the process. The difference in density of gas and liquid results in a difference in velocity $\Delta \mathrm{V}$ and a steady Bernoulli pressure difference $\Delta P \approx \rho(\Delta \rho / \rho)^{2} a^{2} \omega^{2}$ that moves the interface perpendicularly to the vibration direction. Indeed the final interface arrangement, almost perpendicular to the vibration direction, corresponds to a minimum of energy when we consider both potential and kinetic energy.

In the configuration of a hot point source, natural convection $(\mathrm{g})$ is revealed in Fig 2.a. Under weightlessness and high amplitude, low frequency vibration (Fig. 2b), the situation is similar to $1 \mathrm{~g}$, except that buoyancy flows are now symmetrical along the vibration direction. When (Fig. 2c) the amplitude of the vibration is reduced and the frequency increased from case (b), a hot layer moves under convection perpendicularly to the vibration direction (Fig. 1c) - in contrast to the previous case (b).

It is also quite interesting to analyse the case of the well-know Rayleigh-Bénard cell configuration where a fluid is maintained between two horizontal plates separated by the thickness $L$. The lower plate is maintained at a hotter temperature than the top plate, with a constant temperature difference $\Delta \mathrm{T}$. A fluid element of size $r$ starts to rise when the typical convective time across the fluid element is shorter than the diffusion time to the surface, that is $r / V<r^{2} / D_{\text {T. }}$ Here $D_{T}$ is the thermal diffusivity and $V$ is the convective velocity. $V$ is given by the well-known Stokes formula $V \approx r^{2} g \Delta \rho / \eta$. $\eta$ is the shear viscosity. The Rayleigh number gives a measure of the ratio of these two diffusive and convective times: $\mathrm{Ra}=g \frac{\alpha \Delta T L^{3}}{v D_{T}}$ where $\alpha\left(=(1 / \rho)(\partial \rho / \partial T)_{\mathrm{P}}\right.$ is the thermal expansion coefficient. Here one has used the relation $\Delta \rho=\alpha \Delta \mathrm{T}$. Convection starts for $\mathrm{Ra}>1700$.

Let us now consider the same configuration without gravity but under vibration. The criterion for buoyancy-driven convection can be replaced by the presence of a Bernoulli - like pressure difference $\Delta \mathrm{P} \sim \rho(\Delta \rho / \rho)^{2} \mathrm{a}^{2} \omega^{2}$. It results in a driving force $r^{2} \Delta \mathrm{P}$. Applying the same procedure used to define the Rayleigh number, another number is obtained, the vibrational Rayleigh number $\operatorname{Rav}=\frac{(\alpha a \omega \Delta T L)^{2}}{2 v D_{T}}$. The most unstable situation is when the vibration is parallel to the plates ( $\mathrm{Rav}$ is of the order of 2000) and vibration perpendicular to the plates corresponds to a stable situation (no convection). 
(a)

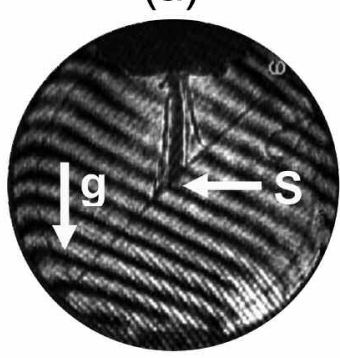

(b)

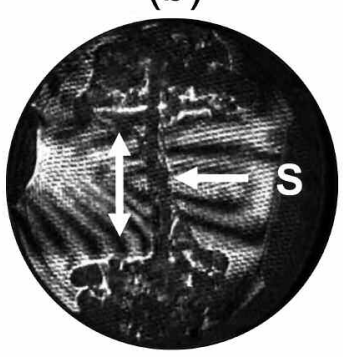

(c)

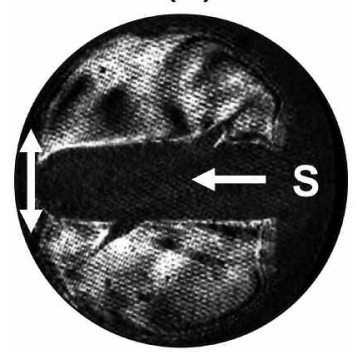

4 Fig. 2: Interferometer images of a sample of supercritical $\mathrm{CO}_{2}$ (12 $\mathrm{mm}$ diameter, $7 \mathrm{~mm}$ thickness) when heated by the point source thermistor S. (a): convection under Earth's gravitational field g. (b) Convection under $64 \mathrm{~mm}$ and $0.2 \mathrm{~Hz}$ large amplitude and low frequency vibration (double arrow) under weightlessness (MIR station, 2000). (c) Convection under lower amplitude (0.8 $\mathrm{mm})$ and higher frequency $(1.6 \mathrm{~Hz})$ vibration (double arrow) (MIR station, 2000).

\section{Phase transition without vibration}

In all the examples given above, the inhomogeneity in density is eventually directed perpendicularly to the vibration direction. Therefore, in some sense, high frequency vibrations can recreate some features of the effects of gravity. However, it is clear that the vibration - and buoyancy - induced flows have different origins. The question that can be now asked is: can this simple and rather naive view be extrapolated to condensation and evaporation? These are quite essential and basic processes since they involve significant heat and mass exchanges and are very non-linear and non-equilibrium phenomena.

The phase transition is concerned at the fundamental level with (i) the nucleation of individual drops or bubbles, not influenced by gravity or vibration flows and (ii) subsequent growth, where drop-drop or bubble-bubble interactions have to be taken into account. Here convective flows really matter. The interactions between drops or bubbles are generally due to the collision and subsequent fusion - i.e. coalescence - of individual bubbles and droplets. Under the Earth's gravitational field, buoyancy that directs bubbles upwards and droplets downwards makes them fuse very quickly, within a kinetics determined by gravity-induced flows. Eventually, after a furious burst of coalescence, the gas-liquid phase separation ends with the gas phase upwards and the liquid phase downwards, separated by a flat meniscus (Fig. 3a).

In space, the effects of gravity are suppressed and the phase transition kinetics is only driven by the haphazard and slower process of collision between droplets or bubbles. Depending on the mean distance between drops or bubbles (which depends on the volume fraction of the minority gas (subscript g) or liquid (subscript l) phase $\phi=v_{\mathrm{g}, \mathrm{I}} /\left(\mathrm{v}_{\mathrm{g}}+\mathrm{v}_{\mathrm{l}}\right)$ as $\left.\phi^{1 / 3}\right)$, the collision can be due to only two processes. For drops or bubbles sufficiently far apart from each other (volume fraction $<30 \%$ ), the collisions are due to Brownian motion (Fig. 3b), with the diameter $D$ of bubbles or drops growing as $D \sim\left(k_{B} T / 6 \pi \eta\right)^{1 / 3} t^{1 / 3}$. Here $T$ is the temperature and $t$ is the time. The Brownian diffusion $\left(\mathrm{t}^{1 / 3}\right)$ growth law corresponds to a very slow evolution.

For a phase transition corresponding to a volume fraction larger than $30 \%$, the drops or bubbles are close together and a new phenomenon becomes important. The hydrodynamic attraction caused by the flow during coalescence is now able to push neighbouring drops close enough to provoke coalescence, thus inducing a kind of chain reaction of drop or bubble fusions. The coalescence of two drops or bubbles leads to fusion with a neighbouring one, and so on. The pattern then looks interconnected (Fig. 3c), with a typical wavelength $\mathrm{Lm}$. The dynamics is at present limited by the flow resulting from coalescence and, for low flow velocity (low Reynolds numbers), growth is linear with time, $L m \approx(\sigma / \eta) t$, where $\sigma$ is the gas-liquid surface tension.

In any case, the kinetics of phase separation, whether limited by Brownian collisions or hydrodynamic interactions, is much slower than when gravity flows stimulate coalescence.

\section{Phase transition under vibration}

In order to investigate under space-like conditions the effect of vibration on evaporation or condensation, experiments have been carried out in a magnetic set-up that creates "artificial" weightlessness on Earth (see Box above fig. 4)). Then "artificial" gravity is added back in, this time in the form of high-frequency $(<50 \mathrm{~Hz})$, low-amplitude $(<0.5 \mathrm{~mm})$ vibrations.

Hydrogen was the fluid under study because of its large diamagnetic susceptibility with respect to other fluids, allowing the magnetic compensation to be achieved with relatively low magnetic field gradients $\left(500 \mathrm{~T} / \mathrm{m}^{2}\right)$. In addition, the phase transition was studied near its critical point $(33 \mathrm{~K})$. The critical point is the end point of the saturation curve and does indeed exhibit very interesting properties. Near the critical point, critical slowing down makes the growth of drops and bubbles very slow. (The gasliquid surface tension $\sigma$ goes to zero whereas viscosity $\eta$ does not vary very much). Also the latent heat goes to zero. In addition, the phase transition can be induced by simply lowering the temperature -thus ensuring no external flows- and within minutes thermal quenches occur (of order on $\mathrm{mK}$ ). A rapid return to

\section{(a)}

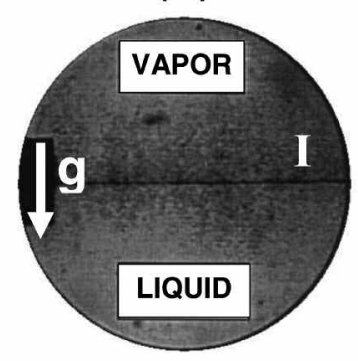

(b)

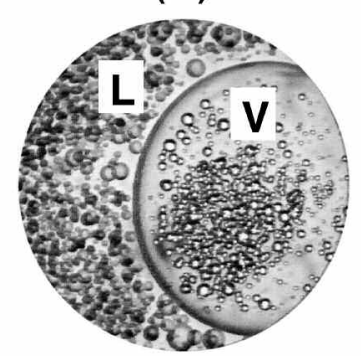

(c)

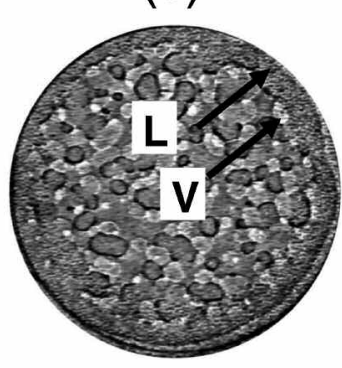

$\Delta$ Fig. 3: Phase separation in a $\mathrm{CO}_{2}$ sample (10 mm diameter, $5 \mathrm{~mm}$ thickness) from above the critical point (supercritical state) to below (gas-liquid equilibrium). (a) Under a gravitational field. I is the gas-liquid interface. Turbulent coalescence of droplets (in the vapour-rich phase $V$ above) and bubbles (in the liquid-rich phase $L$ below) are observed that are induced by buoyancy flows. (b) Under weightlessness, with low $(<30 \%)$ vapour volume fraction. The gas-liquid interface is round. Brownian collisions now drive the process. (c) The same as (b), but with high (>30\%) vapour volume fraction. The pattern is interconnected and the evolution is driven by coalescence-induced capillary flows. 
thermal equilibrium is thus ensured and drop and bubble evolution occurs at strictly constant temperature and pressure (and mean density). In the vicinity of the critical point the results can be expressed in universal, scaled form - but this point will not developed here - see [3]).

What, then, is the effect of vibration? Concerning the interconnected pattern of drops and bubbles (volume fraction larger than 30\%), the results are striking, as shown in Fig. 5. At very early times, the drop and bubble size is so minute that evolution is the same as if there were no vibrations (a). Indeed, for very small domains, the viscous interaction maintains a uniform velocity field, despite the density difference. In other words, the viscous boundary layer $\quad \delta=(\eta / \rho \omega)^{1 / 2}[4]$ is still larger than the typical drop-bubble size Lm. As drops/bubbles grow, Lm exceeds $\delta$. At this moment, a faster evolution is observed (b). This acceleration is followed by the deformation of the gas-liquid interfaces (c) that grow

Recreating the zero-gravity environment of space on Earth

Gradients of magnetic fields, as provided near the end of a coil, can provide a force proportional to the density of diamagnetic and paramagnetic materials. At exact compensation, an artificial gravititational micro-environment is provided for the materials. Most of the studies that are described here were carried out in hydrogen $\left(\mathrm{H}_{2}\right)$ near its critical point $(33 \mathrm{~K}, 1.3 \mathrm{MPa})$ using such a method. Indeed, an advantage is that $\mathrm{H}_{2}$ exhibits a relatively large magnetic susceptibility that enables gravitational forces to be compensated with a realistic superconductor coil $\left(500 \mathrm{~T} / \mathrm{m}^{2}\right.$ near the end of a 10 Tesla coil). Compensation is at the molecular level. It was uniform within $1.5 \%$ of the Earth's acceleration constant in a sample cell of $3 \mathrm{~mm}$ diameter and $3 \mathrm{~mm}$ thickness. Note that it can be shown that a perfect uniform compensation cannot be strictly achieved according to Maxwell's laws [2]).

Vibrating a sample in such high magnetic fields is difficult since the vibrational motion induces eddy currents in metals. For this reason the experimental cell was made with sapphire with the exception of the screws and seals (Fig. 4). The cell was vibrated thanks to a stepping motor and a cam, providing vibration with amplitude $<0.5 \mathrm{~mm}$ and frequency $<50 \mathrm{~Hz}$.

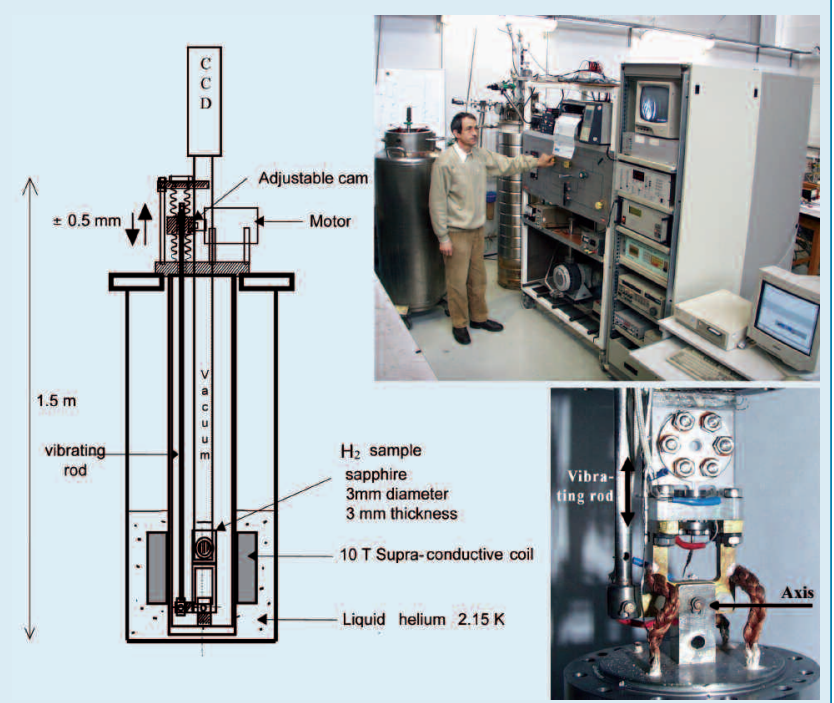

$\Delta$ Fig. 4: Set-up to compensate gravity by magnetic forces (left and top right) and a special vibrating sapphire cell (bottom right). faster in the direction perpendicular to vibration, eventually forming alternate bands of gas and liquid (d). From the simple argument that interfacial energy - and thus interfacial area - has to be a minimum at the end of the process, the steady state should correspond to a single band (bubble) of gas separated by two symmetric liquid bands (drops), as liquid must wet the cell walls. This stage is rarely observed, because the coalescence of alternate bands is impossible without fluctuations; it corresponds to an energy barrier that cannot be overcome with thermal fluctuations. Instead, the sample is pinned in a metastable state that depends on the details of its evolution.

When the volume fraction of the minority phase is lower than $30 \%$, the pattern of evolution is markedly different although retaining some general features of the former evolution. Here too, the effect of vibration remains invisible as long as the size of the growing bubbles does not exceed the viscous boundary layer. The evolution obeys $D \sim t^{1 / 3}$. Then, for $D>\delta$, the bubbles start to "feel" the vibration. It is known [5] that the hydrodynamics of two vibrated inclusions result in repulsion between inclusions in the vibration direction and attraction in the direction perpendicular to vibration. The effect of these repulsive and attractive interactions naturally results in a pattern similar to that shown in Fig. 6, where bubbles organise themselves in periodic, parallel layers. Attraction in the layers increases the bubble coalescence rate and then growth becomes limited by the flow during coalescence (the same as for the interconnected pattern), thus resulting in a linear growth law. For the bubbles that continue to stay between the layers, the growth law remains driven by Brownian coalescence, with the classical $\mathrm{t}^{1 / 3}$ law.

\section{Conclusion}

Returning to the initial question: can high frequency, low amplitude vibrations be used in space as an artificial gravity? The answer is "yes... but" since it depends on the very phenomenon involved. When one deals with thermal convection, interface localisation and even - as mostly reported here - phase separation, vibration can indeed induce mean flows that closely resemble buoyancy. In this sense vibration can really serve as an artificial gravity.

As a matter of fact, small amplitude and high frequency vibration was recently used as an artificial gravity for astronauts. Past studies have shown that vibrating an astronaut's legs and feet helped to prevent muscle decay or bone decalcification, due to the stress induced by vibrations [6].

\section{About the Author}

After receiving an engineering diploma at the Institut d'Optique (Paris), D. Beysens obtained his Ph.D. at Université Paris-6. Director of Research at the CEA he has taken positions at CEASaclay and CEA-Grenoble. He is now working at ESPCI (Paris). $\mathrm{He}$ is interested in phase transitions in fluids, experimenting in space. He loves practicing mountain skiing and hiking - and avoids experiencing free-fall!

\section{Acknowledgements:}

I would like to thank Denis Chatain, engineer at CEA-Grenoble, for his outstanding support, Pierre Evesque, Yves Garrabos and Vadim Nikolayev for essential advice and never-ending encouragement and ESA and CNES for their support..

\section{Further reading}

General on phase transition in fluids:

A. Onuki, Phase transition dynamics (Cambridge University Press, Cambridge, 2002) 
Phase separation under vibration:

D. Beysens, D. Chatain, P. Evesque, Y. Garrabos, High-frequency driven capillary flows speed up the gas-liquid phase transition in zero-gravity conditions, Phys. Rev. Lett. 95,

\section{General on thermo-vibrational phenomena:}

G. Z. Gershuni and D. V. Lyubimov, Thermal vibrational convection (John Wiley \& Sons, Chichester, 1998)

Magnetic compensation of gravity in fluids:

R. Wunenburger, D. Chatain, Y. Garrabos and D. Beysens, Phys. Rev.E 62, 469 (2000)

\section{References}

[1] G. H. Wolf, Z. Physik B, 227(3), 291 (1961);

[2] L. Quettier, H. Felice, A. Mailfert, D. Chatain, D. Beysens., Europhys. Journal of Applied Physics 32, 167 (2005)

[3] D. Beysens, Y. Garrabos, Physica A 281, 361 (2000)

[4] L.D. Landau and E.M. Lifshitz, Fluid Mechanics (Pergamon Press, Elmsford, New York, 1975)

[5] D.V. Lyubimov, T.P. Lyubimova, S.V. Shklyaev, $1^{\text {st }}$ International Symposium on Microgravity Research \& Applications in Physical Sciences and Biotechnology, Sorrento (Italy), Sept. 10-15, 2000, ESA-SP 454, 879 (2001)

[6] The absence of gravity, by the absence of exercise of muscles and stress on bones, cause muscular and bone losses. Slight vibrations (10 $\mu \mathrm{m}$ amplitude, $90 \mathrm{~Hz}$ frequency) transmitted by the life fluids inside the muscles and bones reproduce stresses and, perhaps, could be a solution - at least for bones: astronauts might prevent bone loss by standing on a lightly vibrating plate for 10 to $20 \mathrm{~min}$ utes each day. Held down with the aid of elastic straps, the astronauts could keep working on other tasks while they vibrate. The same therapy might eventually be used to treat some of the millions of people who suffer from bone loss, called osteoporosis, here on Earth. (C.T. Rubin, G. Xu, S. Judex, The anabolic activity of bone tissue, suppressed by disuse, is normalised by brief exposure to extremely low magnitude mechanical stimuli. The FASEB Journal 15, 2225-2229 (2001).

Fig. 5: Evolution of an interconnected pattern of drops and bubbles (volume fraction larger than 30\%). The double arrow represents the vibration. (a): Lm $<\delta$, no vibration influence. (b): $L m \approx \delta$, vibration speeds up the evolution and orientates the drop-bubble interfaces perpendicular to vibration (c) till a steady state is obtained in (d). From (a) to (c): $3 \mathrm{~mm}$ diameter, $3 \mathrm{~mm}$ thickness $\mathrm{H}_{2}$ sample under magnetic compensation of gravity and vibration $20.3 \mathrm{~Hz}$ frequency and $0.3 \mathrm{~mm}$ amplitude, $0.6 \mathrm{mK}$ below the critical point. (d): $10 \mathrm{~mm}$ diameter, $10 \mathrm{~mm}$ thickness $\mathrm{CO}_{2}$ sample in a sounding rocket free fall environment (vibration $42 \mathrm{~Hz}, 0.31 \mathrm{~mm}$, 2 mK below the critical point, ESA Maxus-5 rocket, 2003).

Fig. 6: Evolution of a pattern of bubbles (volume fraction smaller than 30\%). The double arrow represents the vibration. (a): $\operatorname{Lm}<\delta$, no vibration influence, $\mathrm{t}^{1 / 3}$ growth law . (b): $L m>\delta$, vibration induces repulsion (parallel to vibration) and attraction (perpendicular to vibration) that creates a regular pattern of bubbles. In the layer, coalescence is reinforced by the attraction between bubbles, growth speeds up as a linear law. Between the bands, growth is still given by $\mathrm{t}^{1 / 3}$. (c) Steady state. From (a) to (c): $3 \mathrm{~mm}$ diameter, $3 \mathrm{~mm}$ thickness $\mathrm{H}_{2}$ sample under magnetic compensation of gravity and vibration $20.3 \mathrm{~Hz}$ frequency and $0.3 \mathrm{~mm}$ amplitude. (d): $10 \mathrm{~mm}$ diameter, $10 \mathrm{~mm}$ thickness $\mathrm{CO}_{2}$ sample in a sounding rocket free fall environment (vibration $42 \mathrm{~Hz}, 0.31 \mathrm{~mm}, 14 \mathrm{mK}$ below the critical point, ESA Maxus-5 rocket, 2003).

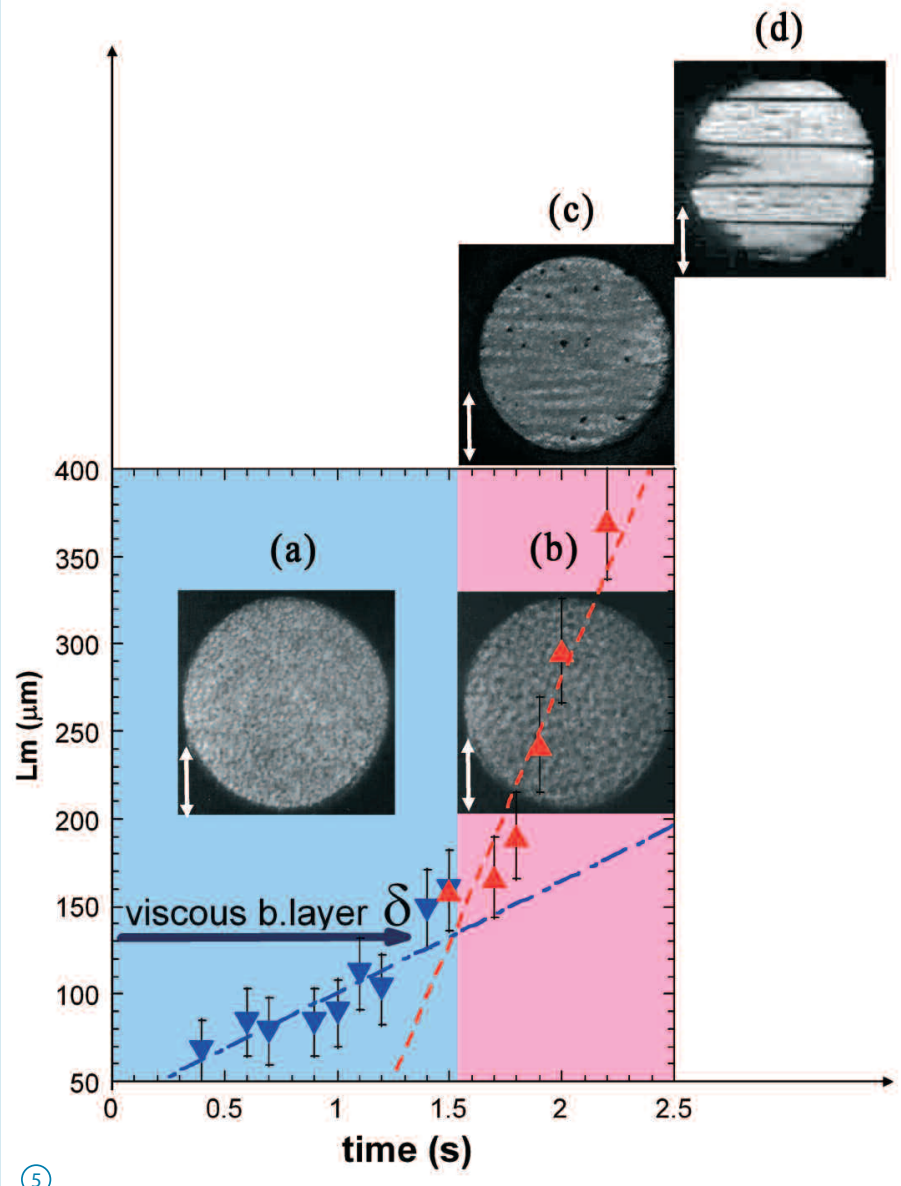

(5)

(6)

(c)
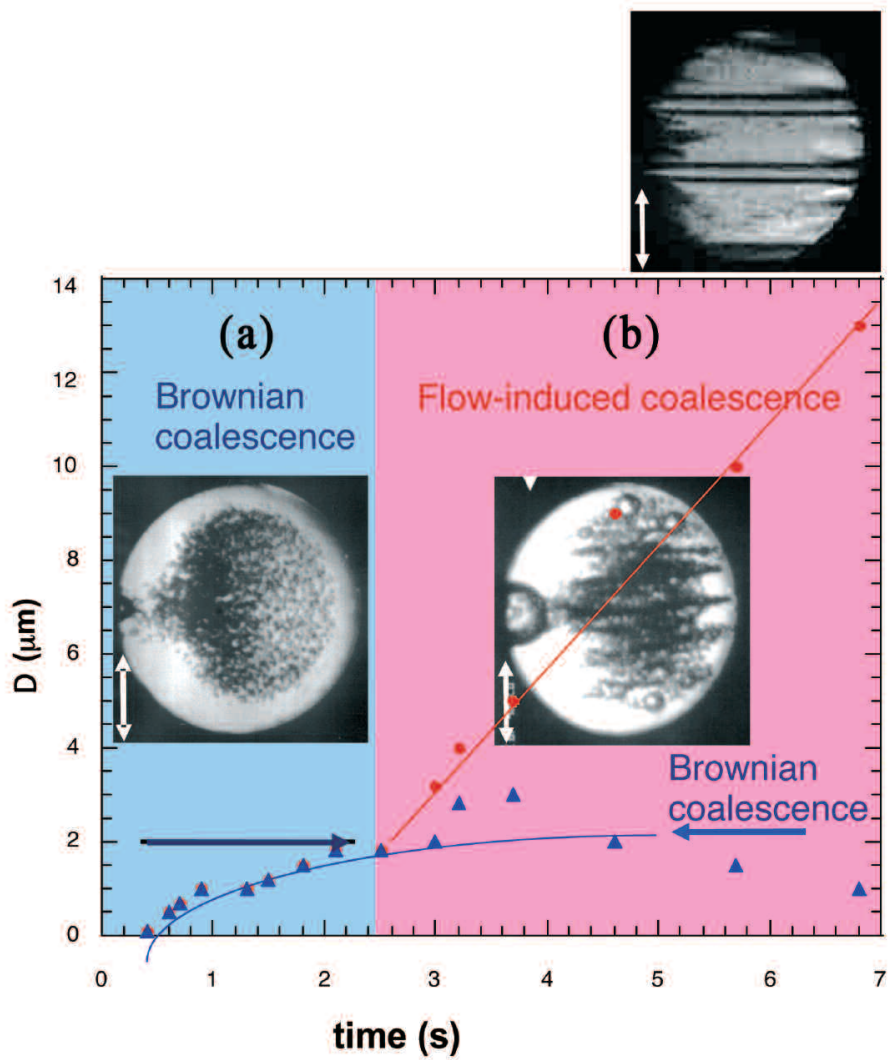